\title{
Post-traumatic stress disorder after mild stroke TIA: psychiatric co-morbidity and symptom cluster distribution
}

\author{
Ho-Yan Yvonne Chun, William N Whiteley, Martin S Dennis, Gillian E Mead, Alan J Carson \\ Centre for Clinical Brain Sciences, University of Edinburgh; Correspondence to hyychun@gmail.com; Twitter @DrYvonneChun; www.task4stroke.org
}

\section{Background}

- Post traumatic stress disorder (PTSD) is thought to occur after mild stroke and transient ischaemic attack (TIA) but has rarely been studied with clinical psychiatric interview

- A better understanding of PTSD symptomology and overlap with other common post-stroke neuropsychiatric sequelae-depression, anxiety, cognitive impairment can help us develop better post-stroke psychological support to patients

Aim

- To describe PTSD symptom cluster distribution at 3 months after mild stroke/TIA according to Diagnostic Statistical Manual-IV criteria

- To assess psychiatric co-morbidity of PTSD, depression and anxiety post-stroke/TIA

\section{Methods}

- Secondary analysis of a prospective cohort of community-based mild stroke and TIA at three months ${ }^{1}$

- Semi-structured diagnostic clinical Interview (SCID-DSM-IV); telephone Montreal Cognitive Assessment (tMOCA)

\section{Results}

- 175 participants: mean age 70; 40\% women; 65\% stroke, and 35\%TIA; median NIH Stroke Score 0, IQR 0-2, median tMOCA 19/22, 17-20

- $11 / 175,6 \%(95 \% \mathrm{Cl} 3-11)$ met the diagnostic criteria for PTSD; A fifth (38/175) of participants had an anxiety disorder

Median tMOCA was 19 in PTSD and non-PTSD cases

\section{All PTSD cases (11/11) were comorbid with an anxiety disorder phobic disorder or generalized anxiety disorder (GAD)}

\begin{tabular}{|c|c|c|c|c|c|}
\hline & $\begin{array}{l}\text { No. of } \\
\text { cases }\end{array}$ & $\begin{array}{l}\text { Sample } \\
\text { frequency }\end{array}$ & Phobic d & GAD & Depressed \\
\hline PTSD & 11 & $6 \%$ & & & \\
\hline Any phobic disorder & 31 & $18 \%$ & 11 & & \\
\hline Any GAD & 20 & $11 \%$ & & & \\
\hline $\begin{array}{l}\text { Depressive episode } \\
\text { (minor/ major) }\end{array}$ & 27 & $15 \%$ & & & \\
\hline
\end{tabular}

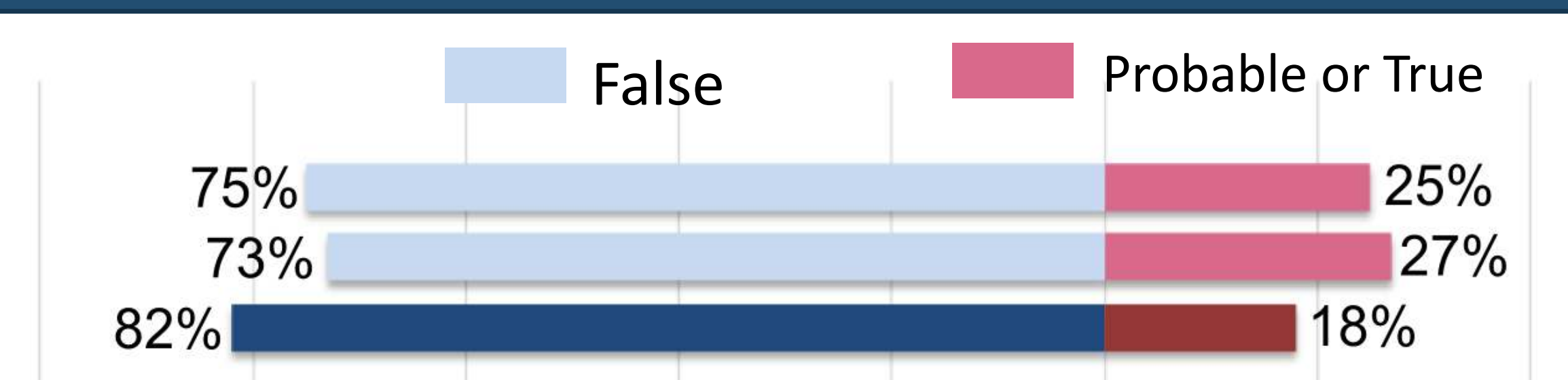
A Criteria: Stressor
A1) The person experienced, witnessed or confronted with an event that involved actual or threatened death
A2) The person's response involved intense fear, helplessness or horror Both A criteria fulfilled
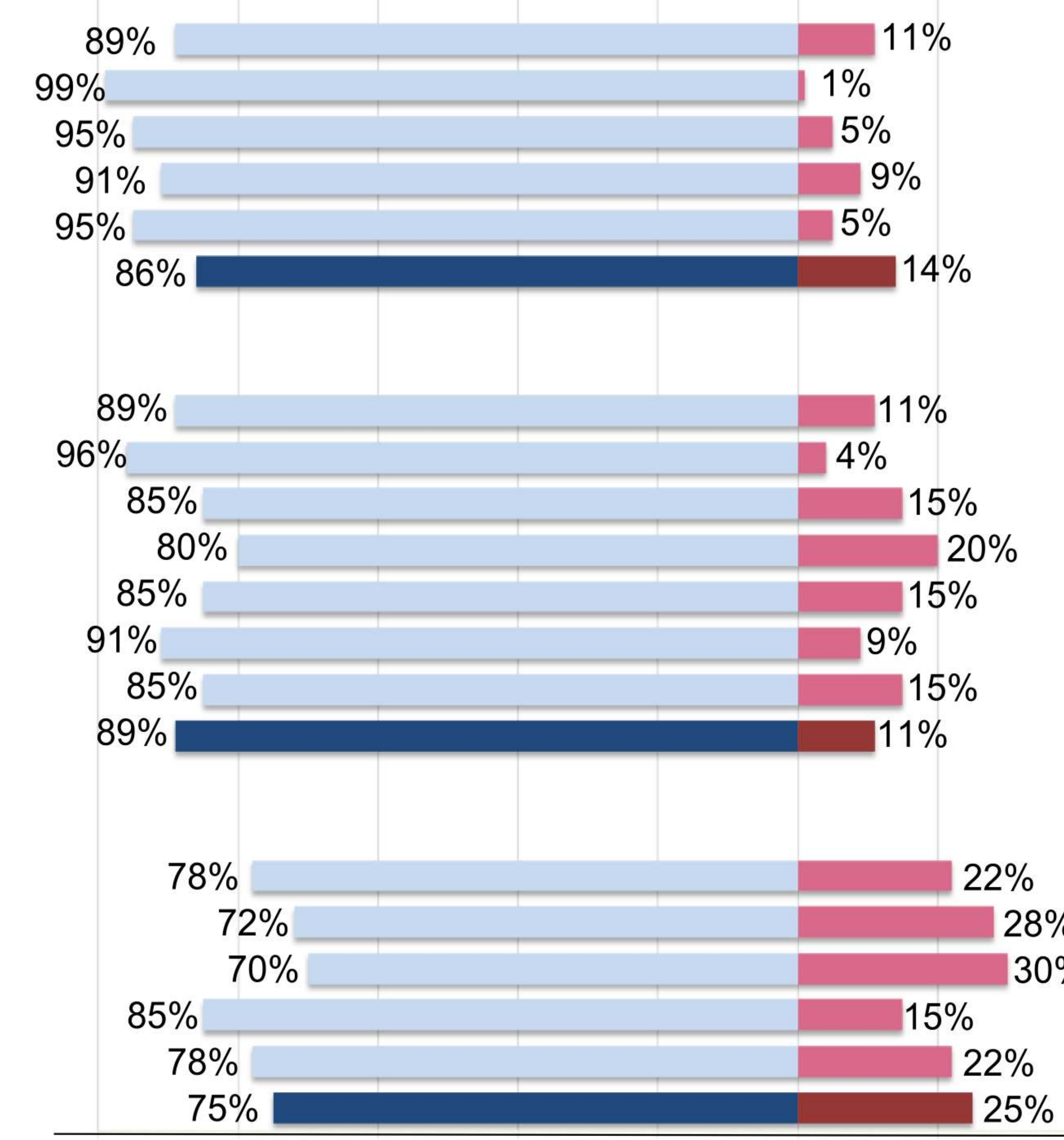

B Criteria: Persistent re-experiencing of stressor event

B1) Recurrent and intrusive distressing recollections of the event, including images, thoughts or perception B2) Recurrent distressing dreams of the event

B3) Acting or feeling as if the traumatic event were recurring (a sense of reliving the experience, illusions, hallucinations, and dissociative flashbacks) B4) Intense psychological distress at exposure to internal or external cues that symbolize or resemble an aspect of the traumatic event B5) Physiological reactivity on exposure to internal or external cues that symbolize or resemble an aspect of the traumatic event At least 1 B criterion fulfilled

C Criteria: Persistent avoidance \& numbing of general responsiveness

C1) Efforts to avoid thoughts, feelings or conversations associated with the trauma

C2) Efforts to avoid activities, places, or people that arouse recollections of the trauma

C3) Inability to recall an important aspect of the trauma

C4) Markedly diminished interest or participation in significant activities

C5) Feeling of detachment or estrangement from others

C6) Restricted range of affect e.g. unable to have loving feelings

C7) Sense of a foreshortened future (does not expect to have a career, marriage, children or a normal lifespan)

At least $3 \mathrm{C}$ criteria fulfilled

D Criteria: Increased arousal

D1) Have you had trouble sleeping?

D2) have you been unusually irritable? What about outbursts of anger?

D3) Have you had trouble concentrating?

D4) Have you been watchful or on guard even when there was no reason to be?

D5) Have you been jumpy or easily startled, like by sudden noises?

At least $2 \mathrm{D}$ criteria fulfilled

$\begin{array}{llllllll}100 & 80 & 60 & 40 & 20 & 0 & 20 & 40\end{array}$

\section{Conclusions \& future directions}

- Clinical diagnosis of PTSD is present in about $6 \%$ at 3 months after mild stroke or TIA, overlapping with depression and anxiety disorders

- PTSD symptoms post-stroke/TIA are common even in those without PTSD diagnosis

- Psychological support for stroke/TIA should consider targeting these common symptom clusters 